\title{
Incidence and familial risk of pleural mesothelioma in Sweden: a national cohort study
}

\author{
Jianguang $\mathrm{Ji}^{1}$, Jan Sundquist ${ }^{1,2}$ and Kristina Sundquist ${ }^{1,2}$
}

Affiliations:

${ }^{1}$ Center for Primary Health Care Research, Lund University, Malmö, Sweden.

${ }^{2}$ Stanford Prevention Research Center, Stanford University School of Medicine, Stanford, CA, USA.

\section{Correspondence:}

Jianguang Ji, Center for Primary Health Care Research, Lund University, CRC, Jan Waldenstroms gata 35, 20502 Malmö, Sweden.

E-mail: Jianguang.jiðmed.lu.se

ABSTRACT Familial clustering of pleural mesothelioma was reported previously, but none of the reports quantified the familial risk of mesothelioma or the association with other cancers. The contributions of shared environmental or genetic factors to the aggregation of mesothelioma were unknown.

We used a number of Swedish registers, including the Swedish Multigeneration Register and the Swedish Cancer Register, to examine the familial risk of mesothelioma in offspring. Standardised incidence ratios (SIRs) were used to calculate the risk. Age standardised incidence rates of mesothelioma were calculated from the Swedish Cancer Registry.

The incidence of mesothelioma reached its peak rate in 2000 and decreased thereafter. Risk of mesothelioma was significantly increased when parents or siblings were diagnosed with mesothelioma, with SIRs of 3.88 (95\% CI 1.01-10.04) and 12.37 (95\% CI 5.89-22.84), respectively. Mesothelioma was associated with kidney (SIR 2.13, 95\% CI 1.16-3.59) and bladder cancers (SIR 2.09, 95\% CI 1.32-3.14) in siblings. No association was found between spouses.

Family history of mesothelioma, including both parental and sibling history, is an important risk factor for mesothelioma. Shared genetic factors may contribute to the observed familial clustering of mesothelioma, but the contribution of shared environmental factors could not be neglected. The association with kidney and bladder cancers calls for further study to explore the underlying mechanisms.

@ERSpublications

Mesothelioma in Sweden began to decrease after 2000; family history is a risk factor for mesothelioma http://ow.ly/4mJemg 


\section{Introduction}

Malignant pleural mesothelioma is a relatively uncommon, aggressive cancer that develops in the pleura, a thin layer of tissue surrounding the lung [1]. Exposure to asbestos is considered the major cause of mesothelioma [2], which may account for more than half of the mesothelioma cases in Sweden and may reach $80 \%$ in many other countries [3-6]. It has been estimated that more than $90 \%$ of asbestos used in Sweden was chrysotile [2]. The use of crocidolite has been prohibited in Sweden since 1975 [2] and from 1982 onwards there has been a general prohibition of the use of other types of asbestos [2]. It is thus interesting to examine whether the incidence of pleural mesothelioma has decreased after the ban of asbestos, and to examine the interval between any fall in the incidence and the timing of the ban to estimate the latency period for the development of mesothelioma.

Besides asbestos exposure, erionite is another established risk factor of mesothelioma [7, 8]. In addition, simian virus $40[9,10]$ may contribute to the development of mesothelioma. Genetic predispositions have been suggested to play an important role in mesothelioma [11, 12], based on the evidence that only a small part of the population exposed to asbestos developed mesothelioma and only certain families in specific villages of Turkey can develop the cancer after exposure to erionite [13]. Familial clustering of mesothelioma has been reported in some previous studies based on a small number of cases [13-16]. However, to the best of our knowledge, no population-based study has been published to quantity the familial risk of mesothelioma. Familial aggregation of mesothelioma may be due to shared environmental factors or genes shared by family members. Some previous studies found that asbestos can be detected in the homes from the working clothes [14] and wives of asbestos-exposed workers showed an excess rate of mortality from mesothelioma [17], suggesting the possible contribution of shared environmental factors on familial aggregation of mesotheliom. In this study, we used a number of Swedish national registers, including the Multigeneration Register, Swedish Cancer Register, Cause of Death Register, and National Population and Housing Census Register, to quantify the familial risk of mesothelioma when the first-degree relatives, including parents and siblings, were diagnosed with mesothelioma and other cancers, and to disentangle the contribution of the shared environmental factors in the families by exploring the relative risk of mesothelioma between the spouses, based on the assumption that spouses could have more intimate contact and thus share more environmental factors than their offspring.

\section{Patients and methods}

This cohort study was approved by the Regional Ethical Review Board of Lund University, Sweden in 2013. This study was carried out by using several nationwide Swedish Registers. The Swedish Cancer Register was founded in 1958 by the National Board of Health and Welfare and has almost complete nationwide coverage [18]. According to instructions from the National Board of Health and Welfare, all physicians in Sweden must report all cases of cancer to the Swedish Cancer Registry; these are called clinical reports. In addition, pathologists must report every cancer diagnosis to the Swedish Cancer Registry based on surgically removed tissues, biopsies, cytological specimens, bone marrow aspirates and autopsies; these are called pathology reports [19-22]. The majority of cancer cases were notified twice from separate reports, guaranteeing a high accuracy rate at the national level. A four-digit diagnosis code according to the seventh revision of the International Classification of Diseases (ICD-7) has been used in the Swedish Cancer Register.

The Multigeneration Register [23], which was created and maintained by Statistics Sweden, includes all children born in Sweden in 1932 and later (maximum 80 years old at 2012), and their siblings and biological parents. More than 14.4 million individuals (living and deceased) in more than 3.8 million families are included in the Multigeneration Register. The data on family relationship have an almost complete coverage, with the exception of some individuals who were born during the 1930s and died before 1991. Although this small group of offspring with missing links to parents has a negligible effect on the estimate of familial risk, we limited the present study to offspring whose parents were known in order to eliminate the possibility of bias. All the individuals from the Multigeneration Register, including both children and parents, were linked to the Swedish Cancer Registry by using the unique individual national registration number.

Additional linkages were made to the National Population and Housing Census [24] to obtain information on individual-level characteristics, such as year of birth, gender and socioeconomic status; to the Cause of Death Register to identify date of death; and to the Emigration Registry to identify date of emigration. All linkages were performed using individual national identification numbers, which were replaced with serial numbers in order to preserve anonymity.

\section{Outcome variable}

The Swedish Cancer Register recorded both the sites and histological types of cancer. Pleural mesothelioma was defined by the ICD-7 code 1622 together with histological diagnosis code 776 . Only the first primary 
pleural mesothelioma was considered in the present study. The incidence rate was standardised according to the age distribution of the European population in 2000.

\section{Predictive variable}

Familial history of cancer was defined by parental and sibling history separately. Other variables included gender and age at diagnosis, which was categorised into 5-year groups. The whole follow-up time was divided into 5-year intervals. Socioeconomic status was recorded according to occupation, divided into six groups based on the Swedish socioeconomic classification by Statistics Sweden: farmer, blue-collar worker, white-collar worker, professional, self-employed and nonemployed (economically inactive individuals, including unemployed individuals and homemakers). Geographical region of residence was divided into three groups: large city (Stockholm, Gothenburg or Malmö), southern Sweden (excluding the large cities, all of which lie in Southern Sweden) and northern Sweden in order to adjust for the possible regional differences in hospitalisation of mesothelioma. Spouses were identified based on common children in the population aged over 25 years.

\section{Statistical analysis}

Person-years at risk (number of persons at risk multiplied by time at risk) were calculated from the start of follow-up on January 1, 1961 until the diagnosis of cancer or death, emigration, or the end of follow-up (December 31, 2012). Standardised incidence ratios (SIRs) were calculated as the ratio of observed to expected number of cases. SIRs were used to measure the relative risk of mesothelioma in individuals with a sibling or parental history of cancer compared with individuals without a sibling or parental history of cancer. Similar calculations were performed separately for spouses. The expected number of cases was calculated for age (5-year groups), sex, interval (5-year groups), geographical region and socioeconomic status-specific standard incidence rates derived from individuals lacking an affected family member. For each type of familial analysis, a specific reference population was used. For calculation of sibling and parental risks, the cohort method was used as described in detail by HemminKi et al. [25]. In the cohort method all siblings in families with two or more affected siblings contributed cases and they were compared with single-case families using the described person-year calculation, which is practically independent of the number of siblings. The 95\% confidence intervals were calculated assuming a Poisson distribution and were adjusted for dependence between the sibling pairs. Data values were accurate to two decimal places. All analyses were performed using SAS version 9.2 (SAS Institute, Cary, NC, USA).

\section{Results}

From the Swedish Cancer Register, a total of 3527 individuals were diagnosed with pleural mesothelioma between 1961 and 2012 with a median age at diagnosis of 69 years. Male patients (2922 cases) outnumbered female patients (605 cases). For those patients with a parental or sibling history of mesothelioma, the median age at diagnosis was 58. Among them, 13 familial cases were males and one was a female. In figure 1 we can see that the incidence of mesothelioma in males increased gradually since 1961, and reached its peak rate in 2000 and decreased thereafter. The trends for females showed a pattern similar to males, but the changes in rates were more moderate.

FIGURE 1 Temporal trend of mesothelioma in Sweden between 1961 and 2012.

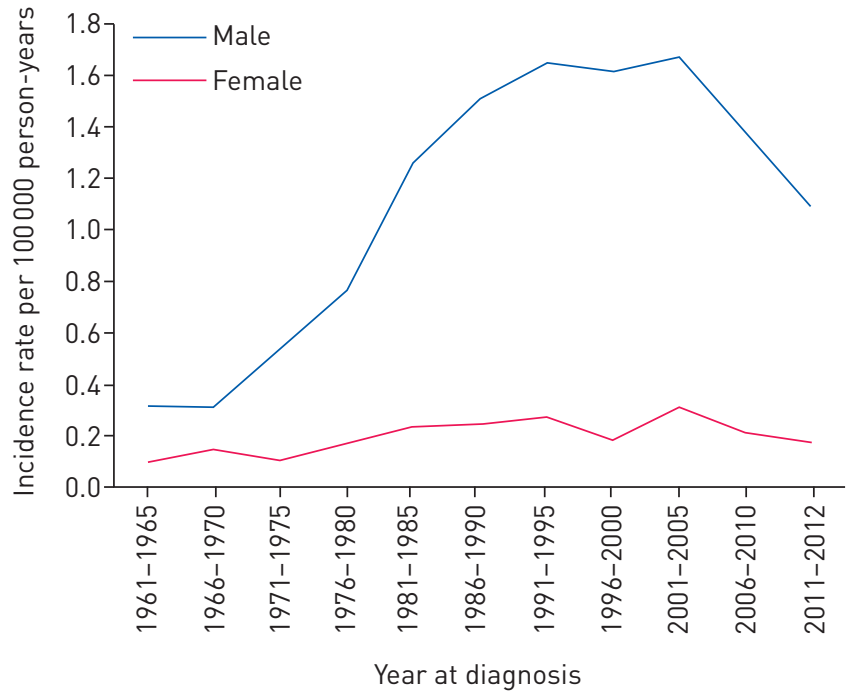


The risk of mesothelioma in individuals with a relative affected with cancer is shown in table 1 . Only those cancer sites with at least five mesothelioma cases are listed. Familial risk of mesothelioma was significantly increased when both parents and siblings were diagnosed with mesothelioma, with SIRs of 3.88 (95\% CI 1.01-10.04) and 12.37 (95\% CI 5.89-22.84) separately. In addition, we found that individuals with a sibling affected with kidney (SIR 2.13) and bladder cancer (SIR 2.09) had an increased risk of mesothelioma.

As the incidence of mesothelioma reached its peak rate in 2000 due to the ban on asbestos, we further examined whether the observed familial risk in table 1 could be varied by the time at diagnosis of mesothelioma (table 2). For mesothelioma diagnosed up to 2000, the sibling SIR was 9.82 and it was 13.90 for mesothelioma diagnosed after that. However, parental risk of mesothelioma was not significant because of a limited number of cases. The incidence of mesothelioma was somewhat higher for those diagnosed up to 2000 with an affected sibling with bladder cancer and for those diagnosed in 2001 or after with an affected sibling with kidney cancer. However, the SIRs stratified by age at diagnosis in table 2 overlap each other due to the large confidence intervals.

To assess the extent to which environmental factors contributed to the observed familial association in table 1, we calculated SIRs for individuals whose spouses were diagnosed with mesothelioma (table 3). The risk of mesothelioma, kidney and bladder cancers was not increased when their spouses were diagnosed with mesothelioma. An increased risk of breast and skin (squamous cell) cancers was found in the wives when their husbands were diagnosed with mesothelioma, and the risk of stomach cancer was increased in the husbands when their wives were diagnosed with mesothelioma.

\section{Discussion}

In this population-based nationwide cohort study, we found that the incidence of mesothelioma reached its peak rate in 2000 in Sweden and decreased thereafter. In addition, we found that the risk of mesothelioma was significantly increased when their siblings or parents were diagnosed with mesothelioma. Additional associations were noted for siblings with kidney and bladder cancers. However, no association was observed between spouses.

TABLE 1 Risk of mesothelioma in offspring when first-degree relatives were diagnosed with cancer

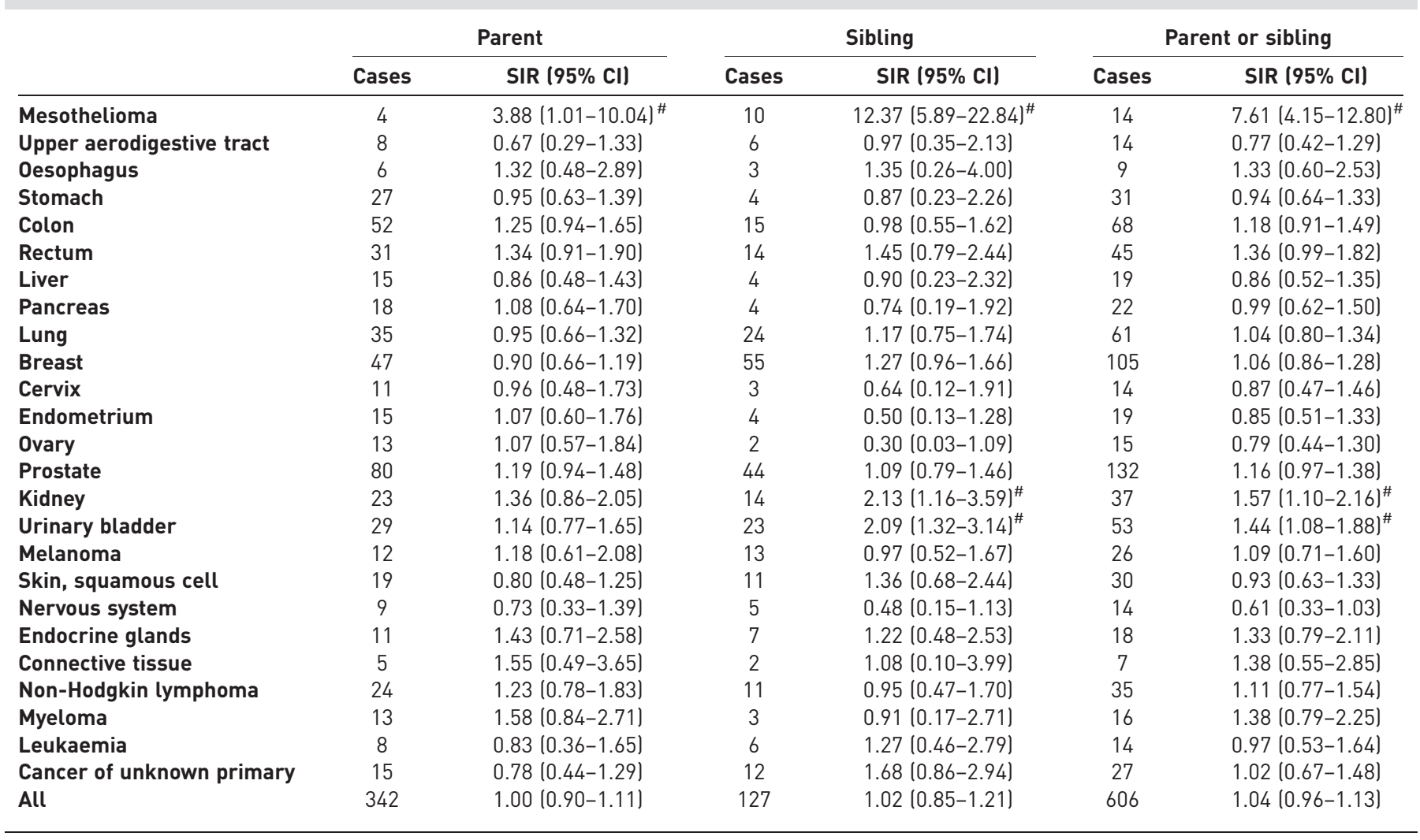

Data are presented as $n$, unless otherwise indicated. SIR: standardised incidence ratio. ${ }^{\#}$ : $p<0.05$. 
TABLE 2 Risk of mesothelioma in offspring by diagnosis period when first-degree relatives were diagnosed with cancer

\begin{tabular}{|c|c|c|c|c|c|c|}
\hline & \multicolumn{2}{|r|}{ Parent } & \multicolumn{2}{|r|}{ Sibling } & \multicolumn{2}{|c|}{ Parent or sibling } \\
\hline & Cases & SIR $(95 \% \mathrm{CI})$ & Cases & SIR $(95 \% \mathrm{CI})$ & Cases & SIR $(95 \% \mathrm{CI})$ \\
\hline \multicolumn{7}{|l|}{ Up to 2000} \\
\hline Mesothelioma & 2 & $5.72(0.54-21.02)$ & 3 & $9.82(1.85-29.06)^{\#}$ & 5 & $7.62(2.41-17.93)^{\#}$ \\
\hline Kidney & 7 & $1.18(0.47-2.45)$ & 1 & $0.42(0.00-2.43)$ & 8 & $0.96(0.41-1.90)$ \\
\hline Urinary bladder & 8 & $0.91(0.39-1.79)$ & 11 & $2.73(1.35-4.90)$ & 19 & $1.46(0.88-2.28)$ \\
\hline \multicolumn{7}{|l|}{2001 and 2012} \\
\hline Mesothelioma & 2 & $2.93(0.28-10.79)$ & 7 & $13.90(5.51-28.80)^{\#}$ & 9 & $7.59(3.44-14.47)^{\#}$ \\
\hline Kidney & 16 & $1.46(0.83-2.38)$ & 13 & $3.10(1.64-5.31)^{\#}$ & 29 & $1.90(1.27-2.74)^{\#}$ \\
\hline Urinary bladder & 21 & $1.27(0.79-1.95)$ & 12 & $1.72(0.88-3.01)$ & 34 & $1.43(0.99-2.00)$ \\
\hline
\end{tabular}

Data are presented as $n$, unless otherwise indicated. SIR: standardised incidence ratio. ${ }^{*}: p<0.05$.

The incidence of pleural mesothelioma has increased greatly since the 1960s in Sweden, especially for men, mainly because of the use of asbestos. Diagnosis procedures for mesothelioma may have changed over the period of study, but are likely to have been a minor factor in the increased incidence, because the observed increase was largely confined to men. In Sweden, the ban of asbestos in the mid-1970s and early 1980s has received great attention $[24,26,27]$. Our data clearly showed that the incidence of mesothelioma reached its peak rate around 2000, which was in line with the previous estimation [24, 26, 27]. The potential latency period of mesothelioma after exposure to asbestos from the current data would appear to be around 20 years.

Although familial aggregation of mesothelioma has been reported previously [13-16], most publications were based on case reports without quantifying the relative risk of familial mesothelioma. The current study

TABLE 3 Cancer risk when spouses were diagnosed with mesothelioma

\begin{tabular}{|c|c|c|c|c|}
\hline & \multicolumn{2}{|c|}{ Husband with mesothelioma } & \multicolumn{2}{|c|}{ Wife with mesothelioma } \\
\hline & Cases & $\operatorname{SIR}(95 \% \mathrm{CI})$ & Cases & $\operatorname{SIR}(95 \% \mathrm{CI})$ \\
\hline Mesothelioma & 1 & $2.12(0.00-12.18)$ & 1 & $2.31(0.00-13.26)$ \\
\hline Upper aerodigestive tract & 3 & $0.45(0.09-1.34)$ & 1 & $0.29(0.00-1.66)$ \\
\hline Oesophagus & 1 & $0.42(0.00-2.39)$ & 2 & $1.56(0.15-5.73)$ \\
\hline Stomach & 13 & $0.96(0.51-1.65)$ & 11 & $2.08(1.03-3.73)^{\#}$ \\
\hline Colon & 45 & $1.03(0.75-1.37)$ & 8 & $0.99(0.42-1.96)$ \\
\hline Rectum & 19 & $0.96(0.58-1.50)$ & 6 & $1.11(0.40-2.44)$ \\
\hline Liver & 18 & $1.19(0.70-1.88)$ & 1 & $0.37(0.00-2.11)$ \\
\hline Pancreas & 19 & $1.34(0.81-2.10)$ & 3 & $0.96(0.18-2.83)$ \\
\hline Lung & 35 & $1.13(0.79-1.57)$ & 11 & $0.98(0.48-1.75)$ \\
\hline Breast & 177 & $1.17(1.01-1.36)^{\#}$ & 0 & \\
\hline Cervix & 14 & $0.74(0.41-1.25)$ & 0 & \\
\hline Endometrium & 32 & $0.93(0.63-1.31)$ & 0 & \\
\hline Ovary & 30 & $1.13(0.76-1.62)$ & 0 & \\
\hline Prostate & 0 & & 34 & $0.99(0.69-1.39)$ \\
\hline Kidney & 14 & $1.06(0.58-1.79)$ & 8 & $2.11(0.90-4.18)$ \\
\hline Urinary bladder & 16 & $1.23(0.70-2.00)$ & 14 & $1.69(0.92-2.84)$ \\
\hline Melanoma & 25 & $1.28(0.83-1.89)$ & 1 & $0.30(0.00-1.71)$ \\
\hline Skin, squamous cell & 32 & $1.47(1.00-2.07)^{\#}$ & 5 & $0.91(0.29-2.14)$ \\
\hline Nervous system & 22 & $1.29(0.80-1.95)$ & 1 & $0.37(0.00-2.11)$ \\
\hline Endocrine glands & 19 & $1.45(0.87-2.26)$ & 2 & $1.90(0.18-6.98)$ \\
\hline Connective tissue & 3 & $0.93(0.17-2.74)$ & 0 & \\
\hline Non-Hodgkin lymphoma & 15 & $0.75(0.42-1.24)$ & 7 & $1.42(0.56-2.95)$ \\
\hline Myeloma & 9 & $1.27(0.58-2.42)$ & 2 & $1.16(0.11-4.26)$ \\
\hline Leukaemia & 6 & $0.64(0.23-1.40)$ & 1 & $0.50(0.00-2.87)$ \\
\hline Cancer of unknown primary & 21 & $1.02(0.63-1.57)$ & 5 & $1.55(0.49-3.65)$ \\
\hline All & 603 & $1.08(1.00-1.18)^{\#}$ & 124 & $1.07(0.89-1.28)$ \\
\hline
\end{tabular}


found that the incidence of mesothelioma was increased around four times for those with an affected parents and increased around 12 times for those with an affected siblings. A few potential factors could explain the higher sibling risk as compared with parental risk. One possible factor leading to the higher sibling relative risk is that the familial relative risk could be age-dependent. In this study, the maximal age for the affected siblings could be under 80 years, whereas the parents used to determine offspring risks may be older. A previous study showed that familial relative risks decline with age [28] and it is thus reasonable that the observed sibling relative risks were higher than offspring risks. Another possible factor is that siblings may share more lifestyle factors compared with parental-offspring pairs, especially for shared environmental factors during childhood [29], which may play an important role in the development of cancer in later life [30, 31]. In addition, the higher sibling risk may be due to autosomal recessive inheritance [25], but such knowledge on mesothelioma is limited. Familial aggregation of mesothelioma may be due to shared environmental factors or genes shared by family members. Our data cannot distinguish between the contributions of inheritability or shared environmental factors, or their interactions, to the observed familial aggregation, because we had no information about asbestos exposure, which is the main strongest risk factor for pleural mesothelioma. Asbestos workers might go home with asbestos dust because of a lack of proper industrial hygiene, thus their family members could be exposed to asbestos (para-occupational exposure), leading to increased familial risk of mesothelioma. To explore the contribution of para-occupational exposure on the observed association, we studied the association between spouses, based on the assumption that spouses could have even more relatively intimate contact as compared with their offspring. We found a nonsignificant association of mesothelioma between spouses, suggesting that shared environmental factors, such as para-occupational exposure to asbestos, might play a minor role in the current findings. However, we cannot neglect the contribution of shared environmental factors based on the current study. Further studies are needed to explore the environmental contribution on the familial aggregation of mesothelioma. Some genetic studies have found that gene polymorphisms in glutathione $S$-transferase M1 [32, 33], $N$-acetyl-transferase 2 [34] and BRCA1-associated protein 1 (BAP1) $[35,36]$ were highly associated with mesothelioma, suggesting that the observed associations may be contributed somewhat by shared genetic factors between family members.

Associations with discordant cancer sites were also tested in the current study. Kidney and bladder cancers in siblings showed a significant association with mesothelioma. However, it should be noted that these could represent chance findings resulting from the large number of associations we examined. Biologic plausibility and consistency with previous findings might be used to exclude chance associations. As no parent-offspring aggregation was found for kidney and bladder cancers, we should be cautious of these observations. However, BAP1 polymorphisms have been found to be associated with both mesothelioma and kidney cancer [37], which may partly explain the observed association.

The advantages of the present study include the identification of cancer patients from a nationwide population database and the completeness of follow-up of patients. All the data were retrieved from nationwide databases, guaranteeing reliable estimation of familial risks because the data on family relationships and cancers were obtained from registered sources with almost complete coverage. In addition, many confounding factors, including age, gender, socioeconomic status and region of residence, were adjusted in the analyses. One limitation of this study is that we had no information about asbestos exposures. However, we have stratified the familial risk by time at diagnosis and the results were largely consistent, suggesting that asbestos exposures may contribute a small part of the observed familial aggregation of mesothelioma.

In summary, the incidence of mesothelioma reached its peak rate after the ban of asbestos in Sweden and began to decrease thereafter. Familial risk of mesothelioma was significantly increased when parents or siblings were diagnosed with mesothelioma, but there was no association between spouses. Shared genetic factors may contribute to the observed familial clustering of mesothelioma, but the contribution of shared environmental factors could not be neglected. In addition, the association with kidney and bladder cancers calls for further study to explore the potential mechanisms.

\section{References}

$1 \quad$ Robinson BW, Lake RA. Advances in malignant mesothelioma. N Engl J Med 2005; 353: 1591-1603.

2 Jarvholm B, Englund A, Albin M. Pleural mesothelioma in Sweden: an analysis of the incidence according to the use of asbestos. Occup Environ Med 1999; 56: 110-113.

3 Pairon JC, Orlowski E, Iwatsubo Y, et al. Pleural mesothelioma and exposure to asbestos: evaluation from work histories and analysis of asbestos bodies in bronchoalveolar lavage fluid or lung tissue in 131 patients. Occup Environ Med 1994; 51: 244-249.

4 Tuomi T, Huuskonen MS, Tammilehto L, et al. Occupational exposure to asbestos as evaluated from work histories and analysis of lung tissues from patients with mesothelioma. Br J Ind Med 1991; 48: 48-52.

5 Lacourt A, Gramond C, Rolland P, et al. Occupational and non-occupational attributable risk of asbestos exposure for malignant pleural mesothelioma. Thorax 2014; 69: 532-539. 
6 Rake C, Gilham C, Hatch J, et al. Occupational, domestic and environmental mesothelioma risks in the British population: a case-control study. Br J Cancer 2009; 100: 1175-1183.

7 Assis L, Isoldi MC. The development of MM is strongly correlated with exposure to asbestos and erionite, as well as to simian virus 40. J Bras Pneumol 2014; 40: 586-587.

8 Ortega-Guerrero MA, Carrasco-Nunez G, Barragan-Campos $\mathrm{H}$, et al. High incidence of lung cancer and malignant mesothelioma linked to erionite fibre exposure in a rural community in Central Mexico. Occup Environ Med 2015; 72: 216-218.

9 Comar M, Zanotta N, Pesel G, et al. Asbestos and SV40 in malignant pleural mesothelioma from a hyperendemic area of north-eastern Italy. Tumori 2012; 98: 210-214.

10 Mazzoni E, Corallini A, Cristaudo A, et al. High prevalence of serum antibodies reacting with simian virus 40 capsid protein mimotopes in patients affected by malignant pleural mesothelioma. Proc Natl Acad Sci USA 2012; 109: $18066-18071$

11 Tunesi S, Ferrante D, Mirabelli D, et al. Gene-asbestos interaction in malignant pleural mesothelioma susceptibility. Carcinogenesis 2015; 36: 1129-1135.

12 Vianna NJ, Polan AK. Non-occupational exposure to asbestos and malignant mesothelioma in females. Lancet 1978; 1: 1061-1063.

13 Roushdy-Hammady I, Siegel J, Emri S, et al. Genetic-susceptibility factor and malignant mesothelioma in the Cappadocian region of Turkey. Lancet 2001; 357: 444-445.

14 de Klerk N, Alfonso H, Olsen N, et al. Familial aggregation of malignant mesothelioma in former workers and residents of Wittenoom, Western Australia. Int J Cancer 2013; 132: 1423-1428.

15 Ribeiro C, Campelos S, Moura CS, et al. Well-differentiated papillary mesothelioma: clustering in a Portuguese family with a germline BAP1 mutation. Ann Oncol 2013; 24: 2147-2150.

16 You B, Blandin S, Geriniere L, et al. Mesotheliomes familiaux: une composante genetique associee aux risques environnementaux? [Family mesotheliomas: genetic interaction with environmental carcinogenic exposure?]. Bull Cancer 2007; 94: 705-710.

17 Ferrante D, Bertolotti M, Todesco A, et al. Cancer mortality and incidence of mesothelioma in a cohort of wives of asbestos workers in Casale Monferrato, Italy. Environ Health Perspect 2007; 115: 1401-1405.

18 Ji J, Sundquist K, Sundquist J, et al. Comparability of cancer identification among Death Registry, Cancer Registry and Hospital Discharge Registry. Int J Cancer 2012; 131: 2085-2093.

19 Ji J, Hemminki K. Familial risk for esophageal cancer: an updated epidemiologic study from Sweden. Clin Gastroenterol Hepatol 2006; 4: 840-845.

20 Ji J, Eng C, Hemminki K. Familial risk for soft tissue tumors: a nation-wide epidemiological study from Sweden. J Cancer Res Clin Oncol 2008; 134: 617-624.

21 Ji J, Hemminki K. Familial blood vessel tumors and subsequent cancers. Ann Oncol 2007; 18: 1260-1267.

22 Ji J, Hemminki K. Familial risk for histology-specific bone cancers: an updated study in Sweden. Eur J Cancer 2006; 42: 2343-2349.

23 Calling S, Ji J, Sundquist J, et al. Shared and non-shared familial susceptibility of coronary heart disease, ischemic stroke, peripheral artery disease and aortic disease. Int J Cardiol 2013; 168: 2844-2850.

24 Jarvholm B, Burdorf A. Emerging evidence that the ban on asbestos use is reducing the occurrence of pleural mesothelioma in Sweden. Scand J Public Health 2015; 43: 875-881.

25 Hemminki K, Vaittinen P, Dong C, et al. Sibling risks in cancer: clues to recessive or X-linked genes? Br J Cancer 2001; 84: 388-391.

26 Hemminki K, Hussain S. Mesothelioma incidence has leveled off in Sweden. Int J Cancer 2008; 122: 1200-1201.

27 Hemminki K, Li X. Mesothelioma incidence seems to have leveled off in Sweden. Int J Cancer 2003; 103: 145-146.

28 Kharazmi E, Fallah M, Sundquist K, et al. Familial risk of early and late onset cancer: nationwide prospective cohort study. BMJ 2012; 345: e8076.

29 Sundquist K, Sundquist J, Ji J. Contribution of shared environmental factors to familial aggregation of common cancers: an adoption study in Sweden. Eur J Cancer Prev 2015; 24: 162-164.

30 Mousavi SM, Brandt A, Weires M, et al. Cancer incidence among Iranian immigrants in Sweden and Iranian residents compared to the native Swedish population. Eur J Cancer 2010; 46: 599-605.

31 Hemminki K, Mousavi SM, Brandt A, et al. Histology-specific risks in testicular cancer in immigrants to Sweden. Endocr Relat Cancer 2010; 17: 329-334.

32 Neri M, Ugolini D, Dianzani I, et al. Genetic susceptibility to malignant pleural mesothelioma and other asbestos-associated diseases. Mutat Res 2008; 659: 126-136.

33 Landi S, Gemignani F, Neri M, et al. Polymorphisms of glutathione-S-transferase M1 and manganese superoxide dismutase are associated with the risk of malignant pleural mesothelioma. Int J Cancer 2007; 120: 2739-2743.

34 Betti M, Neri M, Ferrante D, et al. Pooled analysis of NAT2 genotypes as risk factors for asbestos-related malignant mesothelioma. Int J Hyg Environ Health 2009; 212: 322-329.

35 Ohar JA, Cheung M, Talarchek J, et al. Germline BAP1 mutational landscape of asbestos-exposed malignant mesothelioma patients with family history of cancer. Cancer Res 2016; 76: 206-215.

36 McGregor SM, Dunning R, Hyjek E, et al. BAP1 facilitates diagnostic objectivity, classification, and prognostication in malignant pleural mesothelioma. Hum Pathol 2015; 46: 1670-1678.

37 Joseph RW, Kapur P, Serie DJ, et al. Clear cell renal cell carcinoma subtypes identified by BAP1 and PBRM1 expression. J Urol 2016; 195: 180-187. 